\title{
Eternalism And Propositional Multitasking: In Defence Of The Operator Argument
}

\author{
Draft *
}

\begin{abstract}
It is widely held that propositions perform a plethora of theoretical roles. They are believed to be the semantic values of sentences in contexts, the objects of attitudes, the contents of illocutionary acts, the referents of 'that'-clauses, and the primary bearers of truth. This assumption is often combined with the claim that propositions have their truth-values eternally. Following Kaplan's and Lewis's Operator Argument, I argue that the compositional semantic values of sentences do not correspond to eternal propositions. Therefore, we cannot hold on to both assumptions at the same time: either we regard the non-eternal entities that realize the compositional role of propositions as fulfilling the remaining theoretical roles, or we abandon the assumption that there is a unique realizer. The Operator Argument has recently come under attack, mainly for its intensional assumptions. However, rejecting these assumptions is not a sufficient defense of eternal propositions as compositional semantic values of sentences. Firstly, we can give a generalized version of the Operator Argument that seems independent of the contested assumptions. Secondly, the extensional alternative to the intensional framework does not allow us to retain eternal propositions as unique semantic values either.
\end{abstract}

Keywords: Propositions, Semantic Values, Eternalism, Temporalism, Operator Argument, Compositionality, Sententiality.

\section{Introduction}

What is a proposition? Is it the compositional semantic value of a sentence, or is it the object of belief and other attitudes? Is it what we ascribe to others in attitude reports, or is it what we assert when we utter a sentence? Maybe propositions are the primary bearers of truth and modal properties, or perhaps they are the nodes of logical relations. These roles may not exclude each other, and indeed philosophical orthodoxy has it that propositions are all of the above. ${ }^{1}$ I will

\footnotetext{
* For helpful discussion and comments I would like to thank Albert Atkin, Rachael Briggs, David Chalmers, Adrian Currie, John Cusbert, Jonathan Farrell, Mark Jago, Jeffrey King, Daniel Nolan, Peter Pagin, Brian Rabern, Jonathan Schaffer, Wolfgang Schwarz, Alex Skiles, and Jonathan Tapsell.

1 See e.g. (Salmon and Soames, 1988, Introduction), (Stalnaker, 1999, p. 36), (King, 2003, pp. 195-196), (King, 2007, pp. 1-3), (McGrath, 2007), (Cappelen and
}

(C) 2011 Kluwer Academic Publishers. Printed in the Netherlands. 
label the assumption that there are entities, propositions, that play the above theoretical roles "Propositional Multitasking".

Propositional Multitasking is appealing, as there are obvious interconnections among the foregoing roles. Having a unique realizer would provide us with the prospect of a simple and unified picture of how the different roles mesh. However, things may not be quite that simple. It may turn out that there are different realizers for different roles, or even that the roles impose incompatible demands on their respective realizers, so that no single kind of entity could meet all of them at once. Even then, we would want a story of how the different realizers are related. While such a story would be more complex, there does not seem to be a principled reason why it should be unavailable.

The assumption that there is a unique realizer for the different roles is often paired with a claim about its nature. The standard view is that propositions are entities whose truth-value does not vary across time. Propositions are true or false eternally. Let us label this claim "Eternalism".

In this paper I argue that eternal propositions are unsuitable candidates to play the role of compositional semantic values of sentences in contexts. If that is true, one might either take the non-eternal entities that in fact perform the compositional role to also carry out the further work propositions are supposed to do, i.e give up Eternalism in general. Or, alternatively, one might hold on to the claim that the remaining propositional roles are realized by eternal propositions, but abandon Propositional Multitasking.

The case against eternalist semantic values is based on the so-called "Operator Argument" by David Kaplan (1989) and David Lewis (1980). This argument has recently come under attack from Jeffrey King (2003, 2006) and Herman Cappelen and John Hawthorne (2009), precisely because it endangers the joint acceptance of Propositional Multitasking and Eternalism. However, we can distill an argument from Kaplan and Lewis's considerations which is largely immune to those attacks. Moreover, on the suggested alternative to Kaplan and Lewis's framework, eternal propositions cannot be the only compositional semantic values of sentences either. We need non-eternal semantic values whether we accept the intensional framework of Kaplan and Lewis, or whether we opt for its extensional alternative. Hence, the point remains: we have to give up either Eternalism or Propositional Multitasking.

Hawthorne, 2009, p. 1), (Schaffer, MS, p. 2). Not all of these authors explicitly endorse the entire list. 


\section{Propositional Multitasking}

Before looking at why one might find Propositional Multitasking plausible, let us first state the claim explicitly:

Propositional Multitasking. There are entities, propositions, that realize all of the following theoretical roles:

1. Compositional semantic values of sentences in contexts.

2. Objects of attitudes.

3. Contents of illocutionary acts.

4. Referents of 'that'-clauses (in attitude ascriptions).

5. Primary bearers of truth and modal properties.

If we combine Propositional Multitasking (PM) with Eternalism, we get different versions of Eternalism: Eternalism about the compositional semantic values of sentences, Eternalism about the objects of attitudes, etc. Here, I am going to argue that Eternalism about the compositional semantic values of sentences is false. Since (PM), i.e. the assumption that the realizer of the compositional role performs further theoretical work, is part of the received view, the significance of this result reaches well beyond the realm of semantics. The proponent of $(P M)$ has to give up Eternalism for the additional roles of propositions as well. However, as the argument is based on the first role of propositions only, its consequences hold equally for weaker versions of $(P M)$. It is not essential that propositions play all the mentioned roles, as long as the role of compositional semantic values remains amongst them. We can strengthen the argument by weakening $(P M) .{ }^{2}$ A dedicated Eternalist might alternatively exclude the compositional role as one characteristic of propositions, i.e. abandon $(P M)$ or its weakened siblings. By "compositional semantic values of sentences in contexts", I am referring to the entities that determine (in conjunction with syntactic structure) the semantic values of more complex sentences, i.e. that are the semantic input to the compositional process. ${ }^{3}$

\footnotetext{
${ }^{2}$ For instance, in the dispute with Jeffrey King (2003, 2006), only the first two roles of $(P M)$ really matter.

3 This reading of the first role is common ground in the discussion, see e.g. (King, 2003, p. 197). Instead of the rather longwinded "compositional semantic values of sentences in contexts", I will often just use "semantic values of sentences".
} 
Why is $(P M)$ an attractive claim? Why should we believe that a single kind of entity is able to fulfill all (or most) of the listed roles at the same time? ${ }^{4}$

A first thought might be that $(P M)$ is not merely attractive, but utterly compelling, since $(P M)$ is a conceptual truth. It merely captures how the term "proposition" is used in philosophy.

While it is arguably a conceptual truth that if something plays all the roles, then it is a proposition, it is not a conceptual truth that there are in fact entities that play all the roles. The task of settling the truth of $(P M)$, then, involves examining the different roles one by one, and ascertaining whether all are played by the same kind of entity.

Another consideration that speaks in favor of $(P M)$ is its simplicity. Not only do we require just a single kind of entity, we are furthermore provided with the resources for a unified account of how the different roles connect.

Although simplicity lends support to a theory, its justificatory force is arguably weak and may be outweighed by other theoretical virtues, such as explanatory power. It may very well turn out that the underlying phenomena are more complex than the simple theory assumes. Being simple then becomes being simplistic. When we give an integrated account of the different roles, claiming an identity between the respective realizers is an attractive option, but not the only one available. Another possibility is to postulate weaker relations, such as determination, to connect the different realizers. The semantic value of a sentence might, for instance, be richer than the content of the corresponding assertion, and even though the two are not identical, the former can determine the latter (Lewis, 1980).

A further argument for $(P M)$ derives from more specific considerations relating particular roles. One seductive line of reasoning uses attitude ascriptions as a bridge linking the semantic values of sentences with the objects of attitudes. We use sentences to ascribe attitudes to each other, and compositionality suggests that the semantic value of a complement clause corresponds to the semantic value of the corresponding sentence (when unembedded). ${ }^{5}$ Given that our ascriptions do indeed faithfully report the content of the underlying attitudes, this

\footnotetext{
4 There is some indeterminacy in assessing $(P M)$ : how different must the realizers be to count as different kinds of entities in the relevant sense? This minor unclarity is unproblematic, however, as it is uncontroversial that the three kinds of entities we will be considering here (eternal propositions, temporal propositions, functions from assignments to propositions) would all count as different realizers.

5 Assuming that the complementizer is itself semantically inert; see however (Cresswell, 1985).
} 
suggests in turn that the semantic values of sentences correspond to the objects of attitudes.

There are methodological reservations against a project of this kind, at least if pursued in a naïve manner. In the face of the existence of Mates's puzzle and related phenomena, taking attitude reports at face value and designing our semantic values accordingly threatens to trivialize the whole semantic enterprise (Mates, 1950). ${ }^{6}$ The putative quotational aspect of attitude ascriptions would then entail that no two expressions that differ orthographically (or phonetically) are strictly synonymous. Even if we put these methodological worries aside, doubts remain whether the above argument establishes an identity between semantic values and objects of attitudes. It has, for instance, been questioned whether the correspondence between the content of an attitude report and the content of the reported attitude is as tight as the argument assumes. Attitude ascriptions might be more coarse-grained than the attitudes themselves (or vice versa). ${ }^{7}$

A related consideration in support of $(P M)$ focuses on the fundamental role of language use: we utter sentences to share our beliefs with each other. Very roughly, the picture is this: I believe that p, and I want you to come to believe that $p$ as well. So, I assert that p by uttering a sentence which means that $\mathrm{p}$. Once more, this picture suggests that there is a close connection between our attitudes, the semantic values of the sentences we use to express them, and the contents of the corresponding utterances.

Our reaction to this line of reasoning is by now familiar. It is indeed undeniable that there is an intimate relation between the mentioned roles. However, it is not obvious that this relation has to be one of identity.

Most certainly, there are further arguments to be found that support $(P M)$. Let us assume for now, however, that even though (PM) is prima facie appealing, the case for it is inconclusive.

\footnotetext{
${ }^{6}$ Mates points out that substitution of intuitively clear cases of synonymous expressions (e.g. "chew" and "masticate") can change the truth-values of belief reports.

7 See e.g. (Richard, 1990), (Bach, 1997), (Schlenker, 1999), (Chalmers, MSb). A mere mismatch in content between ascriptions and attitudes does not in itself undermine $(P M)$, for both contents might be realized by entities of the same kind, e.g. different eternal propositions. However, it is a prima facie possibility that the respective contents correspond to different realizers: attitudes might correspond to temporal propositions, and ascriptions to eternal propositions.
} 


\section{Eternalism}

Eternalism is the view that propositions have their truth-value eternally. ${ }^{8}$ The proposition associated with my present utterance of It is raining in Canberra will be true throughout the world's entire history. It will not change its truth-value merely because it stops raining at a later time.

Temporalism, on the other hand, claims that the proposition's truthvalue varies with the weather. It is true at times of rain and false when the sun is shining. As the rain clouds vanish, so does the proposition's truth. However, Temporalism allows for the truth-values of some propositions to be eternal. Not even the Temporalist believes that the truth of $2+2=4$ is transient.

The arguments of the following sections apply to Eternalism as a thesis about the compositional semantic values of sentences. If propositions are the semantic values of sentences, and propositions are eternal, the semantic values of sentences will be eternal propositions.

Semantical Eternalism. Every sentence in context has as its (unique) compositional semantic value an eternal proposition.

In contrast, we can formulate Temporalism, applied to the compositional role of propositions, as the claim that at least some sentences express temporal propositions.

Semantic Temporalism. Some sentences in context have as their (unique) compositional semantic value a temporal proposition.

Semantic Temporalism might seem like the outright negation of Semantical Eternalism (SE). However, the two are not completely exhaustive alternatives. As I am using the labels here, both (SE) and Semantic Temporalism are committed to the claim that sentences are associated with a single semantic value. An alternative to the two views is to connect sentences with multiple semantic values (or to have them contribute different semantic values to different types of linguistic embeddings). Every sentence might express an eternal proposition as one of its semantic values and further a temporal proposition as an additional value (Richard, 1981). A variant of this strategy is to bind the multiple semantic values together into one complex semantic value

\footnotetext{
8 Among many others, see (Frege, 1918), (Richard, 1981), (Cartwright, 1987).
} 
(Chalmers, MSb). Lastly, the semantic values of sentences may correspond neither to eternal, nor temporal propositions, as understood here. $^{9}$

Some utterances of the sentence It is raining in Canberra are true, some are false. The Eternalist explains this fact by claiming the sentence expresses different eternal propositions at different times, some eternally true, others eternally false. Uttered at $t_{1}$, it expresses the eternal proposition that it is raining in Canberra at $t_{1}$, uttered at $\mathrm{t}_{2}$, it expresses the eternal proposition that it is raining in Canberra at $t_{2}$, etc.

As the truth-value of an eternal proposition does not vary within the same possible world, we can assign truth-values to eternal propositions relative to worlds tout court. Within a possible worlds framework, we can then conceptualize eternal propositions as functions from worlds to truth-values. On a structured proposition model, an eternal proposition is a structured entity that literally contains a certain time.

The Temporalist cannot assign truth-values to propositions merely relative to possible worlds. The truth-value of a temporal proposition is determined only with respect to a world and a time. We can identify temporal propositions with functions from worlds and times to truth-values, or the corresponding time-neutral structured entities. The temporal proposition that is associated with the sentence $I t$ is raining in Canberra will take a world and a time to True iff it is raining in Canberra in that world at that time. The Temporalist can explain the variability of an utterance's truth-value by the variability of the truth-value of the corresponding temporal propositions itself.

\section{The Operator Argument}

\subsection{Kaplan's and Lewis's Argument Against Semantic ETERNALISM}

Both David Kaplan (1989) and David Lewis (1980) have put forward an influential argument against (SE). They point out that eternal propositions are not the right compositional arguments for temporal constructions, whereas temporal propositions are. Since Kaplan and Lewis interpret the relevant temporal constructions in analogy with modal operators and their argument relies on how sentences embed under those operators, it has been labelled the "Operator Argument". Their intensional (or tense-logical) understanding of temporal expressions

\footnotetext{
9 This is arguably the case for the functions from assignments to propositions, introduced in $\S 6$.
} 
contrasts with an extensional interpretation thereof, which analyses these expressions in analogy with quantifiers or pronouns. ${ }^{10}$

Within Kaplan and Lewis's framework the truth-value of a sentence is relative to a context and to an index. The semantic value of a sentence type simpliciter is a function from contexts and indices to truth-values. Semantic values of sentences in contexts are functions from indices to truth-values. Here, we are considering whether eternal or temporal propositions (or some third kind of entity) play the role of compositional semantic values of sentences in contexts. In the current setting, this is the question of which parameters indices must contain, whether they contain just worlds, or worlds and times.

Here is Kaplan's formulation of the Operator Argument:

If we built the time of evaluation into the contents (thus removing time from the circumstances leaving only, say, a possible world history, and making contents specific as to time), it would make no sense to have temporal operators. To put the point another way, if what is said is thought of as incorporating reference to a specific time [...], it is otiose to ask whether what is said would have been true at another time [...]. Temporal operators applied to eternal sentences (those whose contents incorporate a specific time of evaluation) are redundant. (Kaplan 1989, 503)

In a nutshell, the argument against (SE) is the following: If the compositional semantic values of sentences that combine with temporal operators were eternal propositions, all temporal operators would be otiose. However, some temporal operators are not otiose. Hence, semantic values of sentences are not identical to eternal propositions.

Kaplan and Lewis's intensional interpretation of temporal construction is a natural approach. It is intuitive that e.g. It is always the case that is the temporal analogue of the modal operator It is necessary that. Just as modal operators are concerned with the truth-values of their argument sentences at different possible worlds, so temporal operators impose certain restrictions on the truth-values of their arguments at different times. Under the assumption that their compositional arguments are eternal propositions, there is not much interesting work for these operators to do. A presently true eternal proposition will be true at all other times, while a presently false one will be eternally false. Within Kaplan and Lewis's framework, (SE) entails that temporal operators are equivalent to truth-functional operators: either they return the present truth-value of the proposition, or they return its opposite truthvalue. Albeit not strictly speaking "redundant", temporal operators

10 The contrast between the intensional and the extensional approach will be explained in more detail in $\S 6$. 
would be far less interesting than they actually are. There would be no room for operators like It is sometimes the case that, which are not simply truth-functional. Even though on some occasions It is sometimes the case that yields a false complex sentence when combined with a false argument sentence, it can also form true sentences out of presently false ones. In spite of it not presently raining in Canberra, the sentence It is sometimes the case that it is raining in Canberra is nonetheless true.

As a consequence, both Kaplan and Lewis concluded that Semantic Temporalism is true, and that the right semantic values for sentences in contexts are provided by temporal propositions. ${ }^{11}$

\subsection{Objections to the Operator Argument}

Kaplan and Lewis's argument has recently encountered significant opposition. The most prominent objections are presented by King (2003, 2006) and Cappelen and Hawthorne (2009). In particular, King has questioned whether Kaplan and Lewis's intensional assumptions can be upheld in the face of recent trends in linguistics and formal semantics. Here I concentrate on King's objections. This is justified by Cappelen and Hawthorne's argumentative strategy. Their objections against the Operator Argument consist mainly in considering specific sample expressions and demonstrating that one or another of the Operator Argument's premises fails for the given example. However, as the Operator Argument (as well as the argument of the next section) rests on a merely existential claim, i.e. on there being some non-otiose temporal embeddings, it is in principle enough to find one convincing case for which all premises hold. The following considerations are supposed to provide such a case.

My strategy here is not to rebut the objections against the Operator Argument, even though I think this can be done. Even if we agreed that Kaplan and Lewis's argument does not constitute a conclusive case for Semantic Temporalism, it would be wrong to conclude that we could blithely return to (SE). However, Cappelen, Hawthorne and King all accept $(P M)$, and they all want to retain the claim that propositions are eternal. They are thereby committed to (SE). None of them perceives this commitment as a problem. Their idea seems to be that once we have rebutted the inappropriate intensional assumptions of Kaplan and Lewis, we can safely resurrect (SE). King puts it thus:

But if the proper way to treat tenses is not as index shifting sentence operators, then there is no need for temporal coordinates in indices

\footnotetext{
${ }^{11}$ Lewis went further and argued that we need functions not only from worlds and times as semantic values, but that indices should additionally include a location and a standard of precision coordinate.
} 
of evaluation. This, in turn, means that we are no longer forced to hold that $[\ldots]$ semantic values are, or determine, functions from worlds, [...] standards of precision and times to truth-values, as Lewis claimed. At most, we are stuck with the view that [...] semantic values of sentences are, or determine, functions from worlds, [...] and standards of precision to truth-values. (King 2003, 223)

The thought here is that in giving up Kaplan and Lewis's intensional interpretation of tense, we no longer require times in the index. Ignoring other parameters, we can supposedly return to the view that semantic values are just functions from worlds to truth-values, i.e. we can reinstate $(S E)$.

This reaction to the Operator Argument is inappropriate. There is a generalization of the Operator Argument which captures its central insight, while being independent of its allegedly inappropriate intensional presuppositions. It is thereby immune to above criticisms. Moreover, even those who remain unmoved by this are bound to give up (SE), since the extensional alternative to Kaplan and Lewis's intensional account does not allow us to retain (SE) either.

\section{The Substitution Argument Against Semantic Eternalism}

According to the Eternalist, even sentences without an explicit time specification, such as It is raining in Canberra, express eternal propositions. Uttered at $t_{1}$, the sentence expresses the eternal proposition that it is raining in Canberra at $t_{1}$. On a possible worlds conception of propositions or the standard structured account, the sentence will express the same proposition as its explicit counterpart It is raining in Canberra at $t_{1}$. On this assumption, a sentence $S$ uttered at $t_{1}$ is semantically equivalent to the explicit version $S$ at $t_{1}$. I will call the sentence $S$ at $t_{1}$ the eternalization of $S$ (when uttered at $t_{1}$ ). And I will call the pair of $S$ and its eternalization $S$ at $t_{1}$, an eternalization pair. (SE) entails that elements of eternalization pairs have the same semantic value.

Eternalization Pairs. Members of an eternalization pair have the same semantic value - they express the same eternal proposition.

I will argue that the fact that (SE) entails Eternalization Pairs shows that $(S E)$ is wrong.

The substitution argument against (SE) is straightforward. (SE) implies that members of eternalization pairs are synonymous (i.e. have the same semantic value). This further implies that they should be 
interchangeable salva veritate in temporal contexts. However, there are temporal contexts in which we cannot interchange eternalization pairs salva veritate. This observation demonstrates that Eternalization Pairs is wrong, and hence that (SE) cannot be upheld.

We will look at embeddings of eternalization pairs within the temporal construction It is always the case that. As it turns out, interchanging members of such a pair within this context can change the truth-value of the resulting complex sentence. Let us consider occurrences of (1) and (2) on the 22nd of August 2010 at 2:36 p.m.:

(1) It is raining in Canberra.

(2) It is raining in Canberra on the 22nd of August 2010 at 2:36 p.m. ${ }^{12}$ According to (SE), (1) and (2) form an eternalization pair: they both express the eternal proposition that it is raining in Canberra on the 22nd of August 2010 at 2:36 p.m. We can further assume that this proposition is actually true. The commitments of (SE) notwithstanding, (1) and (2) cannot be interchanged salva veritate within the temporal construction It is always the case that. Consider sentences (3) and (4):

(3) It is always the case that it is raining in Canberra.

(4) It is always the case that it is raining in Canberra on the 22nd of August 2010 at 2:36 p.m.

I take it to be a datum that (3) is false, while (4) is not. ${ }^{13}$ (3) states that Canberra is a fairly horrible place, a place where it always rains. (3) is false, since, in fact, it is mostly sunny in Canberra. (4), on the other hand, is true as even though there are many times when it does not rain in Canberra, it is still true at all those times that it is raining on the 22nd of August 2010 at 2:36 p.m. The truth of (4) depends only on what the weather is like at the specified date. Since (3) and (4) differ in truth-value, but are attained by interchanging members of the eternalization pair (1) and (2), Eternalization Pairs is false: (1) and (2) are not semantically equivalent, they do not express the same proposition. This implies that $(S E)$ is wrong. Let us examine the assumptions from which the falsity of ( $S E$ ) derives more closely.

Firstly, I have assumed that there are in fact linguistic contexts in which (1) and (2) embed differently. Demonstrating the existence of a Substitution Failure for eternalization pairs is based on the observation that sentences like (3) and (4) differ in truth-value. The best we can do here is to find relevant examples and examine our intuitions regarding

\footnotetext{
12 Let us parse the time-specification as syntactically simple. Otherwise, we could replace it by a name for the time, or the indexical "now".

13 Admittedly, (4) is somewhat cumbersome. However, it is an acceptable sentence of English.
} 
the truth-values of the involved sentences. I take it as a datum that we judge (3) to be false, while we take (4) to be true.

Secondly, establishing the existence of a substitution failure relies on the syntactic premise that (1) and (2) are indeed literally embedded in the more complex sentences (3) and (4). This presupposes that the embedding construction It is always the case that accepts sentential arguments. Let us label this syntactic premise of the argument Sententiality. ${ }^{14}$

Thirdly, inferring information about the semantic values of (1) and (2) from the truth-value of their more complex embeddings requires a systematic connection between the extensions of the complex sentences (3) and (4) and the semantic values of their syntactic constituents. In other words, we need to assume some notion of Compositionality. It is generally accepted that we can formulate compositionality as a substitution claim: two meaningful expressions that differ by substitution of synonymous expressions only are themselves synonymous (Pagin and Westerståhl, 2010a). A fortiori, two such expressions cannot differ in truth-value (when evaluated at the same context). Even though compositionality has been questioned for specific linguistic contexts, it is not contested that the contexts relevant here are compositional. ${ }^{15}$

Fourthly, appearances to the contrary notwithstanding, all of these assumptions are still compatible with the semantic equivalence (in some sense) of eternalization pairs. As Nathan Salmon (2006) has pointed out, we can hold onto semantic equivalence in the face of our data, if we assume that semantic values are not assigned to expressions simpliciter, but rather to expressions relative to types of linguistic environments. ${ }^{16}$ This opens up the possibility that eternalization pairs have identical semantic values when unembedded, but contribute different values to the compositional process within specific types of embeddings. This option is arguably compatible with a generalized notion of compositionality where the semantic function takes pairs of expressions plus linguistic context types as arguments. Even though this is a viable option in principle, I will, for the time being, close off this route by assuming that a semantics assigns semantic values to expressions irrespective of their

\footnotetext{
14 Compare also (Cappelen and Hawthorne, 2009, pp. 70-71).

15 However, see (Soames, 2011). Still, Soames accepts a generalized notion of compositionality, which I will discuss in the next paragraph.

16 See also (Pagin and Westerståhl, 2010a, section 3.8), (Pagin and Westerståhl, 2010b, section 3.2), (Soames, 2011). This approach goes back to Frege, who claimed that expressions in so-called "ungeraden" contexts refer to their ordinary sense (Frege, 1892). These "occurrences" of sentences, i.e. sentences within certain types of linguistic environments, are to be distinguished from sentences within contexts of use, i.e. sentences as they are uttered in certain types of extralinguistic circumstances.
} 
linguistic context. I will label the corresponding assumption Expression Semantics. This contrasts with an "occurrence-based semantics" (Salmon, 2006). Excluding this option in the present dialectic seems legitimate, since the strategy relies on there being several levels of semantic values beyond extension. This clashes with both (SE) and (PM). Not only would we have non-eternal compositional semantic values for sentences, falsifying ( $S E$ ), but, furthermore, there would already be multiple realizers for the compositional role of propositions, falsifying (PM).

Finally, in close connection to the preceding paragraph, $(S E)$ and $(P M)$ are committed to sentences having only one kind of compositional semantic value. The alternative is to associate sentences (irrespective of their embeddings) with multiple compositional values. Again, this is in principle an attractive option. However, it is likewise incompatible with $(S E)$ and $(P M)$. Hence, in the present context we can presuppose Semantic Monism, i.e. the claim that expressions are associated with a unique semantic value.

Leaving the last two points, Expression Semantics and Semantic Monism, as implicit background assumptions, we can reconstruct the substitution argument against (SE) in the following way:

1. Substitution Failure: There are temporal constructions where substituting one member of an eternalization pair for another changes truth-value (when evaluated at the same context).

2. Sententiality: The relevant temporal constructions take sentential arguments.

3. Compositionality: Two sentences that differ only by substitution of synonyms cannot differ in truth-value (when evaluated at the same context).

4. Conclusion: Not all eternalization pairs have synonymous members. Therefore, Eternalization Pairs and hence (SE) are false.

I take it that all premises of the argument are highly plausible, so that we have a strong case against (SE).

Before proceeding, I want to point out a certain limitation of the argument's scope. Plausibly, the implication from (SE) to Eternalization Pairs holds for the standard, unstructured or structured, theories of propositions. It might fail for certain alternatives, such as the nonreductive account of George Bealer (1998). ${ }^{17}$ Having said that, it should

\footnotetext{
17 However, even if the implication ultimately fails, the purported difference in the eternal propositions associated with an eternalization pair must be one that is
} 
again be noted that the substitution argument constitutes only part of the case against $(S E)$.

\section{Against Extensional Eternalism}

\subsection{Intensional vs. Extensional Accounts}

The substitution argument seems independent of the intensional assumptions the Operator Argument relied upon. It generalizes Kaplan and Lewis's case against (SE). In this section, I want to strengthen the case even further by demonstrating that the extensional alternative to Kaplan and Lewis's intensional framework never offered the prospect to save (SE) to begin with. ${ }^{18}$

Within the extensional framework, tenses and other temporal expressions are analyzed as object language quantifiers, or in analogy with pronouns. The extensionalist postulates tacit time variables in the logical form of object language sentences. Temporal constructions bind these time variables. Hence, the extensional treatment assumes that our object language makes (tacit) reference to times.

An intensional interpretation of temporal expressions, in contrast, does not require reference to times in the object language. Just as the standard interpretation of modal operators does not demand referential devices for possible worlds, we likewise do not require representation of times within the object language for the treatment of temporal expressions. And just as modal operators instantiate modally neutral argument sentences at different possible worlds, so temporal operators ask for temporally neutral arguments and evaluate them at different times. ${ }^{19}$

It is important to notice that the conflict between extensional and intensional treatment is not congruent with the one we are interested in

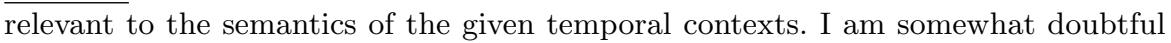
whether it would be a plausible response on behalf of the Eternalist to declare that temporal contexts are hyperintensional contexts, i.e. letting the truth-conditions of temporal embeddings depend on other factors besides the argument's truth-value at different times.

18 The substitution argument and the argument of this section are not completely independent. To establish the conclusion of this section, i.e. that eternal propositions are not the right compositional arguments for temporal quantifiers, we will most likely have to ultimately rely on corresponding substitution failures once more.

19 Nothing in principle, it seems to me, precludes a mixed extensional-intensional account, according to which some temporal expressions function as operators, while others function as quantifiers or pronouns. However, as soon as there are some temporal operators in the language, we will need a temporal coordinate within the index.
} 
here, i.e. the contrast between Semantic Eternalism and Semantic Temporalism. As both Hans Kamp (1968) and Max Cresswell (1990) have shewn, the intensional framework is in principle expressively equivalent to the extensional one. Furthermore, the extensional account is compatible with Semantic Temporalism. If we allow not only for the existence of tacit time variables in the object language, but also for corresponding abstraction operators, the extensionalist can subscribe to the claim that semantic values of sentences are functions from worlds and times to truth-values. ${ }^{20}$

More importantly, however, even on the standard extensional treatment the compositional semantic arguments for tenses and other temporal expressions cannot be eternal propositions! ${ }^{21}$ In $\S 6.3$ we will see why.

\subsection{Definite vs. Indefinite Interpretations of Tense}

I will consider a quantificational implementation of the extensional account. The same considerations apply mutatis mutandis to the pronominal version. King, for instance, is not directly committed to a quantificational interpretation of the extensional framework. In fact, some of the arguments he cites are directed against the claim that the truthconditional contribution of the past tense is equivalent to an existential quantification over times, i.e. against giving it an indefinite interpretation (Partee, 1973).

We have to keep apart the contrast between indefinite and definite interpretations of tense from the distinction between intensional and extensional accounts thereof. The first distinction concerns the semantic contribution of tense: what do the semantic clauses in the metalanguage look like? The second concerns its object language realization: do we have time variables in the logical form of object language sentences or not? The question we are considering right now pertains to the first contrast. It is the question of whether the semantic contribution of e.g. the past tense is existential/indefinite: there is a time in the past, at which $S$ is the case; or whether, on the pronominal/definite understanding, the past tense refers us back to a specific past time: $S$ is the case at $t_{\text {past }}$. Both analyses are in principle compatible with the intensional account.

Several authors point out that most intuitions that speak in favor of a definite, pronominal treatment of the past tense can be accounted for

\footnotetext{
20 See (Heim, 1997), (Kusumoto, 1999). This important fact seems to be often overlooked in the debate. Another proposal to reconcile Semantic Temporalism and the extensional account was put forward by Recanati, see (Recanati, 2007, p. 73-74).

21 A similar point is made in (Almér and Westerståhl, 2010).
} 
within an indefinite, quantificational framework, if we help ourselves to contextually determined quantifier domain restrictions. ${ }^{22}$ Furthermore, as Arnim von Stechow (2009) has argued, even the cases that originally motivated the pronominal treatment are best handled within a quantificational framework. Lastly, the present perfect lends itself even more naturally to an indefinite analysis than the simple past. Therefore, it seems plausible that at least some temporal contexts require an indefinite interpretation. Again, since $(S E)$ makes a claim about the semantic values of all sentences, the existence of some quantificational temporal contexts is all we need for our argument.

\subsection{Temporal Quantification}

On a quantificational reading in its extensional manifestation, the past tense is analyzed as an existential quantifier over times. Let us consider the sentence John laughed. On a simplified analysis, we can assign it the following logical form:

\section{(John) $\exists \mathrm{t} \mathrm{t}<\mathrm{t}^{*} \&$ John laugh-at-t}

The sentence (John) states that there is a time t, which is earlier than time $t^{*}$, and John laughed at that time. If we assign $t^{*}$ to the time of the context, we get appropriate truth conditions for an utterance of John laughed. On this analysis, the embedded sentence contains a time variable and the temporal quantifier binds that variable.

Although this is sometimes neglected, quantificational contexts pose a prima facie problem for compositionality (Janssen, 1997). On a predicate logical treatment, quantifiers like $\forall x$ and $\exists x$ are sentential functors that take open sentences, such as $F x$, as arguments. If we want to give a compositional interpretation of the complex sentence, what semantic value do we have to assign to the open sentence? In predicate logic, the semantic value of an open sentence under an assignment is just a truthvalue. However, this cannot be the compositional argument, as the quantifiers are not truth-functional - we cannot in general calculate the truth-value of the complex sentence $\exists x F x$ from the truth-value of Fx, under a specific assignment. Rather, the compositional semantic value of the open sentence is standardly taken to be a function from assignments to truth-values.

${ }^{22}$ See (Heim, 1997), (Kusumoto, 1999), (Recanati, 2007), (von Stechow, 2009). On the intensional account, we will need an analogue of quantifier domain restrictions for the corresponding temporal operators. Restrictors for temporal operators will function parallel to temporal adverbs like "Yesterday" which are anchored in the utterance time and restrict the time of evaluation to which the tense-operators can shift us. 
The same holds for the semantic arguments of temporal quantifiers. Their arguments cannot be the eternal propositions we get from assigning the free time variable a specific time. Rather, the compositional arguments for temporal quantifiers have to be functions from assignments to eternal propositions. In our example (John), this means that the argument for the temporal quantifier $\exists \mathrm{t}$ will be a function from (ignoring the other elements of the assignments) times to the corresponding eternal proposition that John laughs at that time. Therefore, (John) is true iff there is an assignment which maps a past time to a true eternal proposition, i.e. iff there is a past time at which John laughed. Ignoring again the irrelevant elements of the assignments, compositional semantic values for sentences with free time variables map times to functions from worlds to truth-values. Hence, the compositional values of the extensional account will look quite similar to the intensionalist's temporal propositions. ${ }^{23}$

How are we to understand the role of the assignment function? Is it part of the context, the index, or is it a further and independent argument of the interpretation function? As Thomas Zimmermann (1991) points out, introducing a further argument over and above context and index is a way of identifying a problem, rather than solving it. Interpreting the assignment function as a contextual parameter, on the other hand, has quantificational contexts violating Kaplan's prohibition against monsters. A more promising proposal was put forward by Lewis (1970). The idea is to treat the assignment function as an additional coordinate of the index. On that analysis, temporal expressions, albeit regarded as quantifiers, will still be index-shifting operators, since quantifiers in general shift the assignment coordinate of the index. The quantificational treatment turns out to be a variant of the intensional account.

It should be clear by now that rejecting Kaplan and Lewis's Operator Argument is insufficient as a defense of (SE). The substitution argument against $(S E)$ did not seem to rely on an intensional interpretation of temporal expressions. Moreover, even on the alternative extensional treatment, eternal propositions do not fulfill the relevant compositional role. Rather, that job is done by functions from assignments to eternal propositions; or we can follow Lewis's suggestion and stick to semantic values being functions from indices to truth-values, with indices comprising an assignment coordinate.

\footnotetext{
${ }^{23}$ There might be other ways to handle the semantics of quantification. However, on none of them can temporal quantifiers take the corresponding eternal propositions as arguments.
} 


\section{The Schmentencite Strategy}

Is there not still a way out for the Eternalist? Lewis (1980) pointed out that Semantic Temporalism is not without alternative, calling the alternative route the "schmentencite strategy". He writes:

We can perfectly well build a compositional grammar in which it never happens that sentences are constituents of other sentences, or anything else. [...] In this grammar sentences are the output, but never an intermediate step, of the compositional process. (Lewis 1980, 32)

If we understand the "compositional process" Lewis refers to as the process of semantic composition, we get a semantic version of the schmentencite strategy. Applying this strategy generally, eternal propositions will no longer be compositional semantic values, i.e. entities from which the semantic values of more complex sentences are built. Rather, they will just be non-compositional values, entities that are the final output of the compositional machinery. Leaving everything else untouched, i.e. letting sentences still syntactically embed in larger constructions, we have to ascribe to sentences two kinds of semantic values. We need eternal propositions as non-compositional values, and we also need compositional semantic values. We end up with two kinds of semantic values: input values and output values. By requiring several layers of semantic values, the semantic schmentencite strategy resembles Salmon's occurrence-based semantics and the multiple semantic value strategy, touched upon in $\S 5$. As I noted there, this option clashes with both (SE) and (PM). However, since the semantic schmentencite strategy has only one layer of compositional values, it seems to fare better, since it has only one kind of value realizing the first role of $(P M)$. Still, this role is not fulfilled by eternal propositions, which are output values only. Hence, this version of the schmentencite strategy does not help. Applying the semantic schmentencite strategy selectively, only to the problematic contexts, does not help either. Even though eternal propositions would then enter the compositional machinery some of the times, we would still need non-eternal semantic values for the remaining cases, and hence (SE) and (PM) would both fail once more.

We can supplement the semantic schmentencite strategy with a syntactic component. According to this option, sentences are never syntactically embedded in any other construction, they are always the final upshot of the syntactic composition process. Here is Lewis again:

If we take this course we will need replacements for the sentences hitherto regarded as constituents. The stand-ins will have to be more or less sentence-like. But we will no longer call them sen- 
tences, reserving that title for the output sentences. Let us call them schmentences instead. (Lewis 1980, 32)

What looks like the embedding of a sentence, is now understood as the embedding of a schmentence. Just as eternal propositions are never the input to the semantic composition process, sentences are never the input to the syntactic composition process. We do not need to assign sentences an input value on top of the non-compositional eternal propositions. The resulting picture is this: we can assign sentences eternal propositions as values, because sentences themselves never embed in any other linguistic construction. Since sentences are not part of the syntactic composition process, their semantic values need never play the role of compositional input values.

This strategy does not seem satisfactory as a defense of $(S E)$ and $(P M)$ either. I will discuss its syntactic component in the next section. For now, remember that the claim we are investigating is whether eternal propositions perform the relevant compositional work. The values that our semantics assigns to sentences do nothing of the sort. However, this job still needs to be done. It is now done by the semantic values of schmentences. Let us call their semantic values "schmopositions". Hence, to demonstrate that eternal propositions realize the relevant compositional role the schmentencite has to show that schmopositions correspond to eternal propositions. Is that plausible?

What schmopositions are depends on whether we interpret the relevant constructions in an intensional or in an extensional manner - the schmentencite strategy itself is neutral on this contrast. For the intensionalist, schmopositions are identical to temporal propositions. On the extensional side, the required compositional input values correspond to something like functions from assignments to eternal propositions. ${ }^{24} \mathrm{On}$ neither the intensional nor the extensional option are schmopositions identical to eternal propositions.

The remaining option for the schmentencite is to pursue a selective syntactic strategy. On this approach, sentences do sometimes embed in larger constructions and eternal propositions are compositional input values for some contexts. Hence, eternal propositions play at least part of the compositional role. However, the selective schmentencite concedes to the Temporalist that eternal propositions cannot be the compositional arguments for the contexts under consideration. For those constructions we need non-eternal schmopositions. Having a further kind of input value endangers $(S E)$ and $(P M)$. The only way out is to

\footnotetext{
${ }^{24}$ However, as was pointed out in $\S 6.1$, the connection between intensional/extensional analysis and the corresponding compositional values is not as tight as is usually assumed.
} 
claim that schmentences are not sentence-like syntactic entities. (SE) and $(P M)$ are concerned with the compositional semantic values of sentences. If schmentences are not sentence-like, then schmopositions are not sentential semantic values. The need for non-eternal schmopositions is then, at least nominally, compatible with (SE) and (PM). However, to establish that this is a plausible defense of $(S E)$ and $(P M)$, it is surely insufficient to merely refuse to call the relevant syntactic arguments "sentences" and to label them "schmentences" instead.

If viable, this strategy might provide a response to the substitution argument against (SE). By denying that the relevant temporal constructions take sentential arguments, the opponent denies the Sententiality premise of the argument, and thereby also Substitution Failure. In that case, the complex sentences (3) and (4) cannot be formed by substituting the sentences (1) and (2) into the construction It is always the case that.

Even if this provides a way out for the Eternalist (which I am going to deny), it should be noted that she has already made significant concessions to the Temporalist. The discussion began with the Temporalist pointing out that eternal propositions are not the right compositional arguments for certain temporal constructions. The Eternalist replied that this claim was based on an implausible analysis of the given constructions. However, it became clear that the Temporalist's observation was independent of the contested assumptions, and that the Eternalist had to concede the Temporalist's central point. Now, the discussion shifts from semantics to syntax. The only way for the Eternalist to still uphold ( $S E$ ) is to secure that there are no problematic temporal constructions which are syntactically sentential. In the next section, I show that this putative way out is closed off as well. For one, it is not implausible to regard the syntactic arguments of tenses as sentential. What is more, there are other temporal constructions that uncontroversially take sentential arguments.

\section{Schmentences, Sentences...}

In discussing this attempt to rescue (SE), I will once again concentrate on the quantificational account of tense. On the standard predicatelogical analysis of quantification, the quantifiers $\exists x$ and $\forall x$ are sentential operators that take open sentences, such as Fx, as syntactic arguments. Treating open sentences as sentential entities does not seem to be an accident. Syntactically, the structure it rains at $t$, where $t$ is a free time variable, and the corresponding closed sentence, in which the variable is assigned a specific time it rains at $t_{1}$, do not seem to 
differ in relevant ways - they seem to be of the same syntactic type. Even though there is a difference at one of the terminal nodes of the respective syntactic trees, this difference does not seem to affect the syntactic type of the overarching structures.

On an analysis that is more faithful to the structure of English, ordinary quantifiers are typically analyzed as generalized quantifiers. Generalized quantifiers are not sentential functors. Typically, they take a noun and a verb phrase as arguments. For the case of tense, it is common practice to analyze it as the head of the sentence, which is interpreted as a tense phrase (TP). ${ }^{25}$ Tenses thereby c-command the remaining syntactic structure. Let us consider again the toy example John laughed, under a once more simplified analysis, concentrating on the temporal aspect:

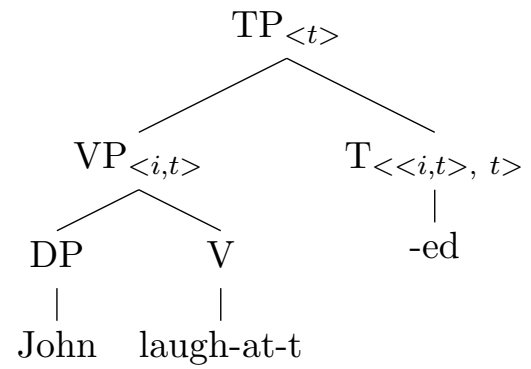

Note that even on the standard extensional analysis (and ignoring worlds), the semantic argument of the past tense is of type $<\mathrm{i}, \mathrm{t}>$. It is a function from times (type $i$ ) to truth-values (type $t$ ), just as Kaplan and Lewis were claiming. Syntactically, tenses have scope over the rest of the tree structure, in our case a VP with an internal subject. Is it legitimate to call these entities "sentential"? They sure look sentential: we have a tenseless subject-predicate structure, exactly as the Temporalist predicted.

However, in a discussion of the Operator Argument's Sententiality premise, Michael Glanzberg (2011) denies that the syntactic arguments of tenses are sentential:

[...] the embedded constituent, a VP or a small clause, is not sentence-like. Though they count as clausal in being predication structures, these are not able to function as matrix clauses, and cannot be asserted. (Glanzberg 2011, 118)

Even though Glanzberg admits that VPs with internal subjects are "clausal", he still thinks that Sententiality fails. However, a few pages earlier Glanzberg states that in assessing Sententiality, "it is the clause, and not the sentence, that is really at issue" (Glanzberg 2011, 115).

${ }^{25}$ See e.g. (Chomsky, 1995), (Heim, 1997), (Kusumoto, 1999), (Radford, 2009), (von Stechow, 2009). 
Glanzberg's denial of Sentententiality might appear somewhat puzzling, since he argues that the crucial question is whether the syntactic argument of tenses are clauses or not and explicitly admits that they are. Maybe Glanzberg's point is that the given VPs are only small clauses, not matrix clauses, and cannot by used by themselves to produce assertoric sentences. However, just as we distinguish propositions in their role as contents of assertions and in their role as compositional semantic values, so we might want to keep apart sentences in their role as vehicles of assertions, and sentences as elements within the syntactic composition process. Here, we are primarily interested in the latter role of sentences.

It is not even clear whether Glanzberg's point about assertibility is right. Irene Heim (1997), for instance, proposes as one option for an extensional analysis of tense to regard temporally neutral predicates of times as the syntactic elements of assertions and to understand temporal reference as established by pragmatic conventions. Put in the predicate-logical paradigm: it is a theoretical option that we assert open sentences with a free time variable, and evaluate these structures conventionally at the time of the context. This would not be an isolated case or an ad hoc suggestion. The same mechanism is operative on a common interpretation of deictic pronouns. Here, the syntactic output is likewise an open sentence with a free (individual) variable, and the assignment to a particular individual is established by pragmatic means (Heim and Kratzer, 1998, Chapter 9). The suggestion gains further plausibility from the standard assumption that logical forms are assigned to expressions irrespective of their extralinguistic context.

The dispute may be verbal and seems to turn on what kind of syntactic entities we are willing to call "sentential". As open sentences seem sentential from a structural perspective and may serve as potential vehicles of assertion, they play some of the characteristic roles of sentences. It may therefore be more than a mere stipulative whim to classify them as "sentential".

Even if the Sententiality premise should fail for tense, it seems very implausible to deny that complex expressions like It is always the case that, It is sometimes the case that, etc. accept sentential or clausal arguments. These constructions are sentential functors par excellence. It is not really controversial that the complementizer phrases they take as arguments are sentential syntactic structures. ${ }^{26}$ Hence, here we have clear examples of sentential constructions whose semantic arguments cannot in general be eternal propositions. I conclude that claiming that

${ }^{26}$ More precisely, the complementizer "that" is the head of the CP. 
there are no non-otiose sentential temporal contexts, and thus denying the Sententiality premise of our argument is not a plausible option.

Remember also that it is part of (PM) that propositions are the referents of 'that'-clauses. To accept that the referents of some 'that'clauses are non-propositional, while denying that these constructions are sentential, would therefore endanger (PM) from another front. ${ }^{27}$

Although this last way out for the Eternalist is closed, the distinction between open and closed sentences seems to open another way to resist the substitution argument against (SE). Even if the extensionalist accepts the Sententiality premise, she might still try to deny Substitution Failure. The idea is that the complex sentence (3) does not really embed sentence (1). Rather, what is embedded in (3) is the open sentence It rains-at- $t$, where $t$ corresponds to a free time variable, and not the closed sentence that corresponds to the unembedded (1), It rains-at- $t_{c}$, where $t_{c}$ is assigned the time of the context.

This response is not unproblematic. As we have seen, it is possible to hold that the open sentence It rains-at- $t$ is the vehicle for the assertion of (1). On that view, (1) is indeed literally embedded in (3). It is also not clear whether the proposed alternative logical form for (1), It rains-at- $t_{c}$, is a viable candidate to begin with, given that logical forms are associated with expression independent of their context of use. However, even if this is a possible reaction to the substitution argument, we have seen in the preceding sections that it does not avoid the need for non-eternal compositional values on the sentential level. Either way, we cannot retain Semantic Eternalism.

\section{Closed Sentence Eternalism}

We might imagine a stubborn Eternalist responding in the following way: 'I agree that, strictly speaking, $(S E)$ is false. However, that the compositional semantic values of open sentences are not identical to eternal propositions is not all that surprising. We could have realized this, for instance, by reflecting on mundane cases of quantification. More importantly, we were really interested in the semantic values of closed sentences all along. And we can still retain (SE) for those and ignore the handful of contexts where we have to deal with open sentences.'

It is true that we could have recognized the inadequacy of (SE) by reflecting on ordinary cases of quantification on a predicate logical

27 Admittedly, this point is compelling only to those who do include the role of referents of 'that'-clauses within $(P M)$. 
rendering. But that does not make our result any less significant. Moreover, to treat constructions involving open sentences as negligible is especially inappropriate for the extensionalist: by extending the extensional treatment to more and more contexts, the need to acknowledge assignment-involving semantic values becomes ever more pressing. King (2003), for instance, proposes to apply the quantificational account to locational constructions, while others have argued that we should even treat ordinary modal constructions extensionally (Schlenker, 2006; Schaffer, 2009). For all these contexts, eternal propositions would be inadequate to fulfill the corresponding compositional job.

What compositional work, then, could remain for eternal propositions? Perhaps, propositional attitude ascriptions could remain? That seems doubtful, as there are well known and very serious problems for a compositional treatment of attitude ascriptions. ${ }^{28}$ It seems unlikely that eternal propositions are universally adequate as compositional arguments for attitudinal contexts. Maybe then, eternal propositions could at least be the arguments for negation, conjunction, and similar functors? On the current picture, this is only part of the story. Negation, conjunction and other connectives will equally operate on the semantic arguments of temporal constructions, i.e. the semantic values of open sentences. Hence, these functors cannot exclusively take eternal propositions as arguments. We would be committed to a widespread ambiguity.

The emerging picture looks unattractive. Rather than clinging to eternal propositions as entities that can merely do part of the compositional work, a more elegant and theoretically simpler option is to uniformly identify compositional semantic values of sentences with functions from assignments to eternal propositions. Closed sentences would then correspond to constant functions that map every assignment to the given eternal proposition, while open sentences would (typically) correspond to non-constant functions. Again, Lewis's proposal to deal with the assignment function is an attractive alternative. Both options would provide us with a unified picture of semantic values and allow us to circumvent postulating an extensive ambiguity for expressions that take both open and closed sentences as arguments.

\footnotetext{
${ }^{28}$ There is Frege's puzzle (Frege, 1892), Mates's puzzle (Mates, 1950), and relevantly, the problem of essential indexicals in attitude ascriptions (von Stechow, 1984). It is also not hard to see that there will be substitution failures for eternalization pairs within doxastic contexts.
} 


\section{Whither Now?}

We have to abandon Semantical Eternalism. Should we then retain Eternalism for the other propositional roles and reject Propositional Multitasking instead? Even though I am skeptical about the ultimate viability of $(P M)$, we should not give it up lightly, especially since there is good reason to reject Eternalism for other roles as well. There are convincing arguments that the objects of (at least some) beliefs are given by temporal propositions. ${ }^{29}$ A similar case, it seems to me, can be made for the contents of illocutionary acts. There is therefore some hope for the Temporalist of retaining $(P M)$.

However, I believe that in the end we will need multiple values (or corresponding complex values) at least for the compositional role of propositions. ${ }^{30}$ Moreover, even the Temporalist might have to bring in assignments to deal with cases of bound pronouns in embedded contexts, such as: Everybody thinks that they are smart. For the Temporalist, the Lewisian treatment of assignments seems especially natural. It is an interesting question whether these assignment-involving values are able to perform any further propositional roles.

\section{Conclusion}

In this paper I have argued that we cannot retain both Eternalism and Propositional Multitasking, since eternal propositions are not apt to play the compositional role of propositions. This result is independent of the intensional assumptions for which the Operator Argument has been criticized. For one, the substitution argument seems to not rely on a specific interpretation of the involved temporal expressions. Moreover, the extensionalist never had the option to identify sentential semantic values of sentences uniformly with eternal propositions to begin with. Within the extensional framework at least part of the compositional job has to be performed by different entities. I have suggested that the most promising strategy for the extensionalist is to identify compositional semantic values of sentences with functions from assignments to propositions or with sets of assignment-containing indices.

\footnotetext{
29 See (Lewis, 1979), (Chalmers, MSa). Lewis's account subsumes beliefs with eternal contents. Hence, the proposal provides the Temporalist with a unified account of the objects of belief.

${ }^{30}$ It may of course turn out that these multiple or complex values are the ultimate realizers of $(P M)$.
} 


\section{References}

Almér, A. and D. Westerståhl: 2010, 'Review of Relativism and Monadic Truth'. Linguistics and Philosophy 33, 37-50.

Bach, K.: 1997, 'Do Belief Reports Report Beliefs'. Pacific Philosophical Quarterly 78, 215-241.

Bealer, G.: 1998, 'Propositions'. Mind 107, 1-32.

Cappelen, H. and J. Hawthorne: 2009, Relativism and Monadic Truth. Oxford: Oxford University Press.

Cartwright, R.: 1987, Philosophical Essays. Cambridge, MA: MIT Press.

Chalmers, D.: MSa, 'Frege's Puzzle and the Objects of Credence'. Forthcoming in Mind.

Chalmers, D.: MSb, 'Propositional Attitudes: A Fregean Account'. Forthcoming in Noûs.

Chomsky, N.: 1995, The Minimalist Program. Cambridge, MA: MIT Press.

Cresswell, M.: 1985, Structured Meanings. Cambridge, MA: MIT Press.

Cresswell, M.: 1990, Entities and indices, Studies in Linguistics and Philosophy. Kluwer.

Frege, G.: 1892, 'Über Sinn und Bedeutung'. Zeitschrift für Philosophie und philosophische Kritik 100, 25-50.

Frege, G.: 1918, 'Der Gedanke'. Beiträge zur Philosophie des deutschen Idealismus 1, 8-77.

Glanzberg, M.: 2011, 'More on Operators and Tense'. Analysis 71, 112-123.

Heim, I.: 1997, 'Lecture Notes on Tense'. MIT.

Heim, I. and A. Kratzer: 1998, Semantics in Generative Grammar. Oxford: Blackwell Publishers.

Janssen, T. M. V.: 1997, 'Compositionality'. In: J. van Benthem and A. ter Meulen (eds.): Handbook of Logic and Language. Amsterdam: Elsevier, pp. 419-473.

Kamp, H.: 1968, 'Tense Logic and the theory of linear orders'. Ph.D. thesis, University of California, Los Angeles.

Kaplan, D.: 1989, 'Demonstratives'. In: J. Almog, J. Perry, and H. Wettstein (eds.): Themes from Kaplan. New York: Oxford University Press.

King, J. C.: 2003, 'Tense, Modality, and Semantic Values'. Philosophical Perspectives 17, 195-245.

King, J. C.: 2007, The Nature and Structure of Content. Oxford University Press.

Kusumoto, K.: 1999, 'Tense in embedded contexts'. Ph.D. thesis, UMass Amherst.

Lewis, D.: 1970, 'General Semantics'. Synthese 22, 18-67.

Lewis, D.: 1979, 'Attitudes de Dicto and de Se'. The Philosophical Review 88, 513-43.

Lewis, D.: 1980, 'Index, Context, and Content'. In: S. Kanger and S. Öhman (eds.): Philosophy and Grammar. Dordrecht: D. Reidel, pp. 79-100.

Mates, B.: 1950, 'Synonymity'. University of California Publications in Philosophy 25, 201-26.

McGrath, M.: 2007, 'Propositions'. Stanford Encyclopedia of Philosophy. http://plato.stanford.edu/entries/propositions/

Pagin, P. and D. Westerståhl: 2010a, 'Compositionality I: Definitions and Variants'. Philosophical Compass 5, 250-264.

Pagin, P. and D. Westerståhl: 2010b, 'Compositionality II: Arguments and Problems'. Philosophical Compass 5, 265-282.

Partee, B. H.: 1973, 'Some Structural Analogies Between Tenses and Pronouns in English'. Journal of Philosophy 70, 601-609. 
Radford, A.: 2009, Analysing English Sentences: A Minimalist Approach. Cambridge: Cambridge University Press.

Recanati, F.: 2007, Perspectival Thought. Oxford: Oxford University Press.

Richard, M.: 1981, 'Temporalism and Eternalism'. Philosophical Studies 39, 1-13.

Richard, M.: 1990, Propositional Attitudes. New York: Cambridge University Press.

Salmon, N.: 2006, 'Terms in Bondage'. Philosophical Issues 16, 263-274.

Salmon, N. and S. Soames (eds.): 1988, Propositions and Attitudes. Oxford: Oxford University Press.

Schaffer, J.: MS, 'Confessions of a Schmentencite'. Unpublished manuscript.

Schlenker, P.: 1999, 'Propositional attitudes and indexicality'. Ph.D. thesis, MIT.

Soames, S.: 2011, 'True At'. Analysis 71, 124-133.

Stalnaker, R. C.: 1999, Context and Content, Oxford Cognitive Science. Oxford: Oxford University Press.

von Stechow, A.: 1984, 'Structured Propositions and Essential Indexicals'. In: F. Landman and F. Veltman (eds.): Varieties of Formal Semantics. Proceedings of the 4th Amsterdam Colloquium, September 1982. Dordrecht: Foris Publications, pp. 384-404.

von Stechow, A.: 2009, 'Tenses in compositional semantics'. In: W. Klein and P. Li (eds.): The Expression of Time, The Expression of Cognitive Categories. Mouton de Gruyter.

Zimmermann, T. E.: 1991, 'Kontextabhängigkeit'. In: A. von Stechow and D. Wunderlich (eds.): Semantik/Semantics: Ein internationales Handbuch der zeitgenössischen Forschung. Berlin/New York: Walter de Gruyter, pp. 156-229. 
"Eternalism and Propositional Multitasking".tex; 21/09/2011; 22:21; p.28 